\title{
Caspase-3 activation in systemic anaplastic large-cell lymphoma
}

\author{
Elias Drakos ${ }^{1}$, George Z Rassidakis ${ }^{1}$, Raymond Lai ${ }^{1}$, Marco Herling ${ }^{1,2}$, Sean L O’Connor ${ }^{3}$, \\ Annette Schmitt-Graeff ${ }^{2}$, Timothy J McDonnell ${ }^{3}$ and L Jeffrey Medeiros ${ }^{1}$ \\ ${ }^{1}$ Department of Hematopathology, The University of Texas MD Anderson Cancer Center, Houston, TX, USA; \\ ${ }^{2}$ The Institute of Pathology, Albert-Ludwigs-University of Freiburg, Germany and ${ }^{3}$ Department of Molecular \\ Pathology, The University of Texas MD Anderson Cancer Center, Houston, TX, USA
}

\begin{abstract}
Anaplastic large-cell lymphoma (ALCL), as currently defined, includes a subset of tumors that have abnormalities of chromosome 2p23 (alk gene) resulting in overexpression of anaplastic lymphoma kinase (ALK). We have previously shown differences in apoptotic rate and expression of apoptosis-related proteins between ALK-positive and ALK-negative ALCL. In this study, we assessed for activated caspase-3 (aC-3), an executioner of apoptotic cell death, in ALCL cell lines and tumors. We used the Karpas 299 and SU-DHL-1 cell lines, and the caspase inhibitors Boc-D-FMK and DEVD-FMK to investigate the role of caspase-3 activation in tumor cell death after treatment with doxorubicin. Cell viability and apoptosis were assessed by trypan blue and Annexin-V methods. A caspase-3 assay was used to evaluate caspase-3 enzymatic activity. Caspase-3 activity was significantly increased in Karpas-299 and SU-DHL-1 cells treated with doxorubicin, but remained as low as control levels with addition of Boc-D-FMK or DEVD-FMK. Expression of aC-3 was also assessed immunohistochemically in 57 ALCL tumors. The mean percentage of aC-3 positive tumor cells was $3.2 \%$ in ALK-positive ALCL compared with $1.2 \%$ in ALK-negative ALCL $(P=0.0003$, Mann-Whitney test), and inversely correlated with BCL-2 expression ( $P=0.01$, Mann-Whitney test). aC-3 expression did not correlate with patient outcome in either the ALK-positive or ALK-negative ALCL groups. In conclusion, doxorubicin-induced cell death of ALK-positive ALCL cells involves caspase-3 activation in vitro. aC-3 levels correlate with ALK expression in ALCL tumors.
\end{abstract}

Modern Pathology (2004) 17,109-116. advance online publication, 5 December 2003; doi:10.1038/modpathol.3800039

Keywords: activated caspase-3; anaplastic large-cell lymphoma; ALK; BCL-2; prognosis

Anaplastic large-cell lymphoma (ALCL), as defined in the recently published World Health Organization (WHO) classification, ${ }^{1}$ includes a subset of cases with 2 p23 locus abnormalities ${ }^{2-6}$ that disrupt the anaplastic lymphoma kinase (alk) gene and result in the overexpression of ALK. The most frequent abnormality in ALCL, the $\mathrm{t}(2 ; 5)(\mathrm{p} 23 ; \mathrm{q} 35)$, encodes the chimeric NPM-ALK (NPM-nucleophosmin) protein ${ }^{7}$ that is known to be oncogenic. ${ }^{8,9}$

It has been reported that patients with ALKpositive ALCL have a better clinical outcome than patients with ALK-negative ALCL, ${ }^{10-12}$ but the

Correspondence: LJ Medeiros, Department of Hematopathology, Box 72, University of Texas MD Anderson Cancer Center, 1515 Holcombe Blvd, Houston, TX 77030, USA.

E-mail: jmedeiro@mail.mdanderson.org

This study was presented in part at the XIth Meeting of the European Association for Hematopathology, May 26-30, 2002, Siena, Italy.

Received 25 June 2003; accepted 14 October 2003; published online 5 December 2003 underlying mechanisms for this survival difference are unknown. Previously, we have shown that ALKpositive tumors lack BCL-2 expression and exhibit a relatively higher apoptotic rate compared with ALKnegative ALCL, ${ }^{13}$ suggesting that tumor cell apoptosis in response to chemotherapy plays a role.

Apoptotic pathways may be involved in ALCL oncogenesis, although the exact mechanisms are not completely understood. ${ }^{14}$ Previous in vitro studies have demonstrated that NPM-ALK is capable of activating the phosphatidylinositol 3-kinase (PI3K)Akt/PKB pathway. ${ }^{15,16}$ Activation of this pathway results in phosphorylation of the BCL-2 family protein $\mathrm{BAD}$, preventing $\mathrm{BAD}$ from binding to the antiapoptotic proteins BCL-2 and BCL-XL and inhibiting their function. As a result, unbound BCL-2 and BCL-XL may promote tumor cell survival. ${ }^{15,16}$ Recently, it has been shown that NPM-ALK also activates the JAK/STAT pathway ${ }^{17-19}$ resulting in downstream antiapoptotic signals.

Caspases are a group of proteases that possess an active cysteine site and cleave proteins at specific 
sites with a tetrapeptide configuration. ${ }^{20}$ Caspases constitute the core of the apoptotic machinery. More than 10 caspases have been identified in humans, either in inactive zymogen or activated protease forms, of which caspase 3 (CPP32) plays a major role. ${ }^{20}$ Apoptotic signals from both extrinsic (cell surface) ${ }^{21}$ and intrinsic (mitochondrial) pathways can activate initiator caspases 8 and 9 leading to proteolytic activation of other effector caspases including cleavage of caspase 3 into two fragments. ${ }^{22}$ These fragments can form a tetramer with two heterodimer subunits, constituting the active form of caspase 3. Activated caspase-3 (aC-3), in turn, cleaves target proteins resulting in apoptotic death. ${ }^{23}$

Caspase-3 in its inactive form, known as procaspase-3 (proC-3), has been detected in the tumor cells of Hodgkin's disease ${ }^{24}$ and non-Hodgkin's lymphomas. ${ }^{25}$ Moreover, proC-3 immunostaining patterns have been correlated with clinical outcome in patients with diffuse large B-cell lymphoma (DLBCL). ${ }^{26}$ Activated caspase-3 is localized in the nucleus and correlates with apoptotic morphology in B-cell non-Hodgkin's lymphomas. ${ }^{27}$

In this study, we investigated the role of caspase-3 activation in ALCL using two well-established ALCL cell lines. Our in vitro data indicate that caspase-3 in ALCL cells is activated after treatment with doxorubicin and that specific caspase- 3 inhibitors can rescue ALCL cells from death. We also assessed for expression of aC-3 and proC-3 in a series of systemic ALCL tumors obtained from previously untreated patients. Our results show significant differences in aC-3 levels between ALKpositive and ALK-negative tumors.

\section{Materials and methods}

\section{Cell Lines}

Two ALCL cell lines were used, Karpas 299 (a gift from Dr M Kadin, Boston, MA, USA) and SU-DHL-1 (DSMZ, Braunschweig, Germany). Both cell lines carry the $t(2 ; 5)$ and overexpress ALK protein. The cell lines were maintained in RPMI 1640 medium supplemented with $1 \%$ nonessential amino acids, $10 \%$ fetal calf serum (GIBCO, Invitrogen Corporation, Grand Island, NY, USA), and 1\% streptomycin-penicillin. Cells were incubated at $37^{\circ} \mathrm{C}$ in a humidified atmosphere containing $5 \% \mathrm{CO}_{2}$.

\section{Induction and Detection of Apoptosis}

To determine apoptosis in ALCL cell lines, SU-DHL-1 and Karpas 299 cells were incubated at a concentration of $7.5 \times 10^{5}$ cells $/ \mathrm{ml}$ with $5 \mu \mathrm{M}$ of doxorubicin in 24-well plates for $20 \mathrm{~h}$. The dosage of doxorubicin was chosen after preliminary experiments that assessed the sensitivity of both cell lines to different concentrations of this agent (data not shown). These cells were also pretreated with Boc-D-FMK, a broadspectrum caspase inhibitor, as well as the caspase-3 selective inhibitor DEVD-FMK (both from Calbiochem, San Diego, CA, USA) at a concentration of $100 \mu \mathrm{M}$ each. Cell viability was evaluated using trypan blue staining in triplicate using a hemocytometer slide and the mean percentage of viable cells was calculated. In addition, Annexin-V staining (BD Biosciences Pharmingen, San Diego, CA, USA), detected by flow cytometry, was used to assess cell death according to the manufacturer's instructions. Briefly, $5 \times 10^{5}$ cells were washed in ice-cold PBS and resuspended in $200 \mu \mathrm{l}$ of binding buffer. Subsequently, cells were incubated with $10 \mu \mathrm{l}$ 7-AAD and $5 \mu \mathrm{l}$ Annexin-V for $15 \mathrm{~min}$ in the dark at room temperature and $10^{4}$ ungated cells were counted using a flow cytometer (FACS Calibur, BectonDickinson). Control cells were included in each set of experiments. These experiments were repeated twice.

\section{Evaluation of Caspase-3 Activity}

Cells were treated with doxorubicin for $6 \mathrm{~h}$. Caspase3 activity was determined by measuring the ability of cell lysates to cleave a fluorescently coupled caspase-3 substrate. Briefly, after induction of apoptosis, cells were harvested, washed with PBS, and resuspended in lysis buffer containing $10 \mathrm{mM}$ HEPES/KOH pH 7.4, 2 mM EDTA, 1 mM DTT, 0.1\% IGEPAL, and protease inhibitors PMSF, antipain, pepstatin A, and leupeptin. ${ }^{28}$ Protein concentrations were then determined using a Bradford assay. ${ }^{29}$ Caspase activity reactions consisted of $100 \mu \mathrm{g}$ of total protein in $100 \mu \mathrm{l}$ of lysis buffer combined with $100 \mu \mathrm{l}$ assay buffer (10 $\mu \mathrm{M}$ DEVD-AMC caspase-3 substrate (Calbiochem, La Jolla, CA, USA), $100 \mathrm{mM}$ HEPES, 10\% Sucrose, 0.1\% CHAPS, $0.1 \mathrm{mM}$ EDTA, $1 \mathrm{mM}$ DTT). Reactions were performed in fluorescent assay 96-well microtiter plates. Kinetic measurements were performed in an HTS 7000 plate reader (Perkin-Elmer, Wellesley, MA, USA) over a 12-h period.

\section{ALCL Tumors}

This group included 57 cases of systemic ALCL (22 ALK + , 35 ALK-) accessioned at The University of Texas MD Anderson Cancer Center and The University of Freiburg, Germany, between 1984 and 2000. All tumor specimens were obtained prior to therapy. The median age of patients with ALKpositive tumors was 29 years compared with 50 years for patients with ALK-negative tumors ( $P=0.0001$, Mann-Whitney test). Patients with ALK-positive ALCL were more frequently women (14/22 vs $8 / 35, P=0.0045$, Fisher's exact test). All other clinical parameters including Ann Arbor stage were comparable as reported previously. ${ }^{13}$ The diagnosis of ALCL was based on morphologic and 
immunohistologic criteria according to the WHO classification. ${ }^{1}$ All tumor specimens were routinely processed, fixed in 10\% buffered formalin and embedded in paraffin. All ALCL cases uniformly expressed CD30 and were negative for B-cell antigens. All T-cell tumors were positive for one or more T-cell antigens; null cases were negative for all T-cell antigens assessed.

\section{Design and Construction of the Tissue Microarray}

A manual tissue microarrayer (Beecher Instruments, Silver Spring, MD, USA) was used to construct a tissue microarray as previously described. ${ }^{30}$ The tissue microarray used included triplicate tumor cores from 38 cases of systemic ALCL, seven cases of primary cutaneous ALCL (not further discussed) and two reactive lymph nodes. All three tumors cores from each case were evaluated. Full tissue sections were used for 19 additional ALCL tumors.

\section{Immunohistochemical Methods}

The immunohistochemical methods have been described previously. ${ }^{13}$ Briefly, tissue sections (3 or $4 \mu \mathrm{m}$ thick) were deparaffinized in xylene and rehydrated in a graded series of ethanols. The antibodies used were specific for: proC-3 (polyclonal, DAKO, Carpinteria, CA, USA; dilution 1:100), aC-3 (clone C92-605, BD Pharmingen, dilution 1:400), ALK-1 (DAKO, dilution 1:30), BCL-2 (DAKO, dilution 1:40), and MIB-1 (Ki-67, Immunotech, Westbrook, ME, USA; dilution 1:120). For all antibodies, heat-induced epitope retrieval was performed. ${ }^{13}$ A three-step biotin-streptavidin-horseradish peroxidase method was used as described elsewhere. Tumor cores from reactive follicular hyperplasia served as internal positive controls for proC-3 and aC-3 immunostaining. ${ }^{25}$

Any cytoplasmic or nuclear staining of ALCL cells for proC3 was considered positive, irrespective of intensity. ProC-3 expression was considered as preserved, reduced or increased when the intensity of staining was similar, weaker or stronger, respectively, when compared with a reactive germinal center. Any nuclear staining of tumor cells for aC-3 was considered positive. Expression levels for aC-3 were determined by counting at least 1000 neoplastic nuclei in each case. For the purpose of survival analysis, we used the median percentage of aC-3 positive tumor cells as a cutoff. The percentage of MIB-1-positive ALCL cells was designated as the proliferation index.

\section{TUNEL Assay}

The apoptotic rate of the ALCL tumors was evaluated using a modified terminal deoxynucleotidyl transferase (TdT)-mediated dUTP nick end- labeling (TUNEL) assay as described previously. ${ }^{13}$ The percentage of TUNEL-positive tumor cell nuclei was designated as the apoptotic rate.

\section{Statistical Analysis}

The nonparametric Spearman's rank correlation coefficient was applied to evaluate the strength of the relationship between aC-3, proliferation index and apoptotic rate. The $\chi^{2}$ and Fisher's exact tests were used to compare the expression of all proteins as groups (positive vs negative) with various clinicopathological parameters. The Mann-Whitney $U$-test was chosen for the nonparametric correlation of aC-3 with ALK expression in ALCL.

Progression-free survival (PFS) was chosen to evaluate the clinical outcome of patients. Overall survival (OS) was also evaluated. Analysis was based on the method of Kaplan and Meier with the Mantel-Cox logrank test. All computations were carried out using the StatView statistical program (Abacus Concepts, Inc., Berkeley, CA, USA).

\section{Results}

\section{Doxorubicin-induced Apoptosis and Activation of Caspase-3 in ALCL Cell Lines}

Using trypan blue staining, the mean percentage of viable SU-DHL-1 cells after treatment with $5 \mu \mathrm{M}$ of doxorubicin decreased from 96 to $38 \%$ at $20 \mathrm{~h}$ (Figure 1a). Addition of the caspase-3 inhibitor DEVD-FMK rescued a substantial proportion of SU-DHL-1 cells, resulting in $74 \%$ viable cells. Incubation of cells with the broad-spectrum caspase inhibitor Boc-D-FMK rescued most cells, resulting in $91 \%$ of viable cells (Figure 1a). Similar results were obtained for Karpas 299 cells (Figure 1b).

These findings were confirmed by the Annexin-V assay (Figure 2) that demonstrated that most SU-DHL-1 and Karpas 299 cells pre-treated with DEVD-FMK remained viable. Pretreatment of cells with Boc-D-FMK also rescued most SU-DHL-1 and Karpas 299 cells from apoptotic cell death.

Caspase-3 activity was assessed in SU-DHL-1 cells treated with doxorubicin for $6 \mathrm{~h}$. The results are illustrated in Figure 3. Incubation of SU-DHL-1 cells with doxorubicin resulted in a significant increase of caspase-3 enzymatic activity, which did not occur in similar incubations after addition of the DEVDFMK or Boc-D-FMK caspase inhibitors (Figure 3).

\section{Expression of aC-3 in ALCL Tumors}

Tumor cells positive for aC-3, with a nuclear staining pattern, were detected in all 57 ALCL tumors assessed (Figure 4). The majority of aC-3 positive tumor nuclei had apoptotic morphologic features. Activated caspase-3 was also present in the cytoplasm of a variable number of histiocytes 


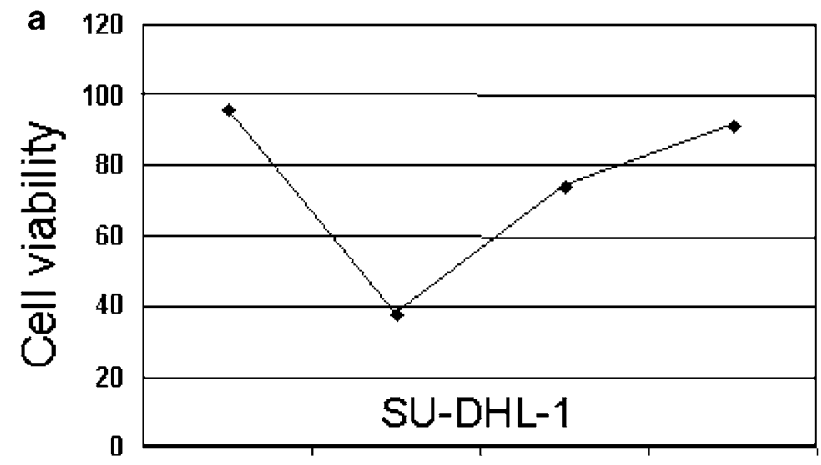

b

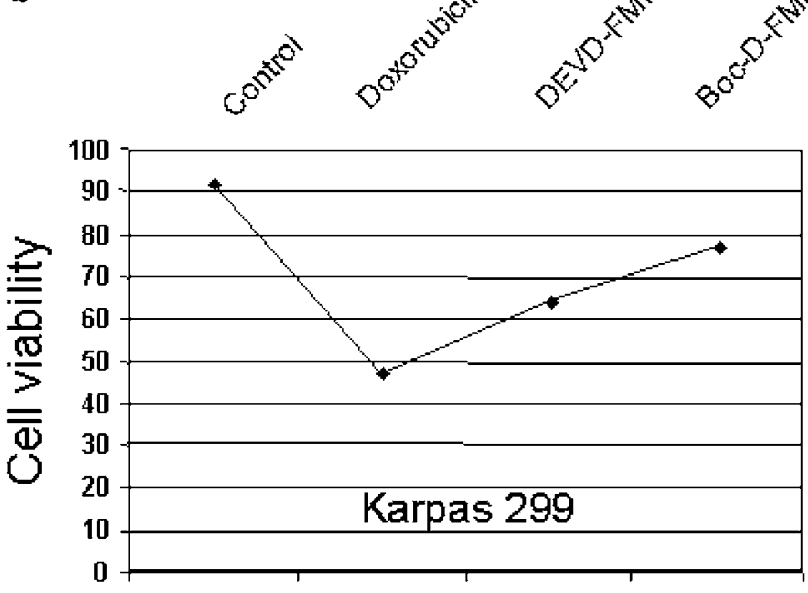

Figure 1 Evaluation of cell viability using trypan blue staining: (a) The mean percentage of viable SU-DHL-1 cells after treatment with $5 \mu \mathrm{M}$ of doxorubicin decreased from 96 to $38 \%$ at $20 \mathrm{~h}$. DEVD-FMK rescued most SU-DHL-1 cells (viable cells $74 \%$ ), whereas Boc-D-FMK rescued almost all SU-DHL-1 cells (viable cells, 91\%). (b) Similarly, treatment of Karpas 299 cells with $5 \mu \mathrm{M}$ of doxorubicin resulted in $47 \%$ cell viability, whereas addition of DEVD-FMK and Boc-D-FMK resulted in 64 and $77 \%$ viable cells, respectively.

containing apoptotic bodies and debris. The percentage of aC-3 positive tumor nuclei in ALCL varied from 0.1 to $7.8 \%$, with a mean of $1.9 \%$ and a median $1.4 \%$. Apoptotic bodies in histiocytes were not included in this percentage.

Correlation of aC-3 levels with clinical features at time of diagnosis showed that aC-3 levels were higher in younger ALCL patients (Spearman's $R=0.41, \quad P=0.0019$ ). Levels of aC-3 were not associated with other clinical and laboratory characteristics including gender, Ann Arbor stage, B-symptoms, bone marrow involvement, anemia, and serum levels of $\beta$-microglobulin, LDH or albumin.

\section{Association of aC-3 Level with ALK and BCL-2 Expression}

Activated caspase-3 levels correlated with ALK expression. The mean percentage of aC-3 positive tumor cells was $3.2 \%$ in ALK-positive ALCL
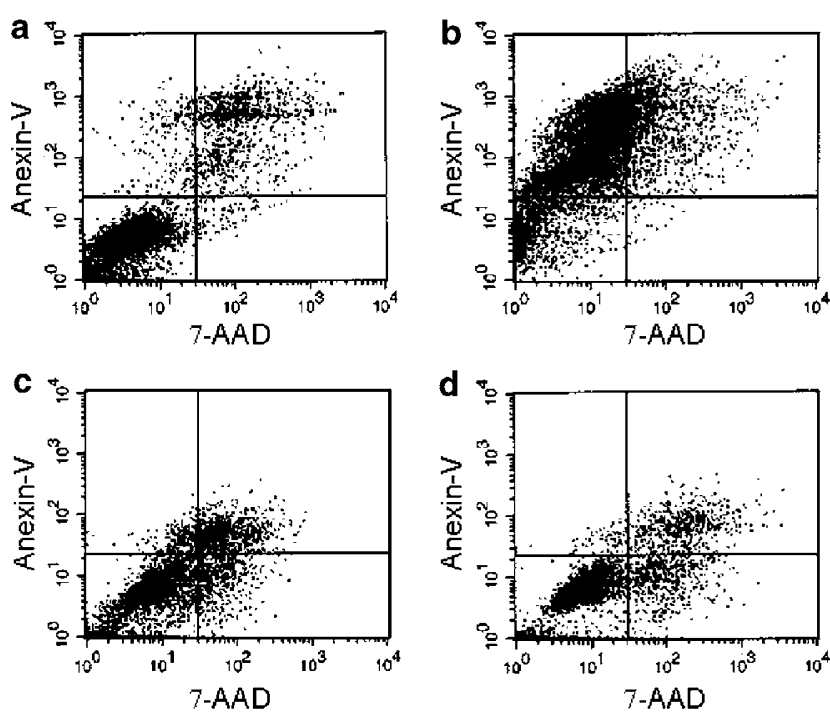

Figure 2 Annexin-V and 7-AAD as detected by flow cytometry: (a) control SU-DHL-1 cells; (b) SU-DHL-1 cells treated with $5 \mu \mathrm{M}$ of doxorubicin; (c) SU-DHL-1 cells pretreated with DEVD-FMK and $5 \mu \mathrm{M}$ of doxorubicin. Most cells remained viable. (d) SU-DHL-1 cells pretreated with Boc-D-FMK and $5 \mu \mathrm{M}$ of doxorubicin. Boc-DFMK rescued almost all SU-DHL1 cells from apoptotic cell death.

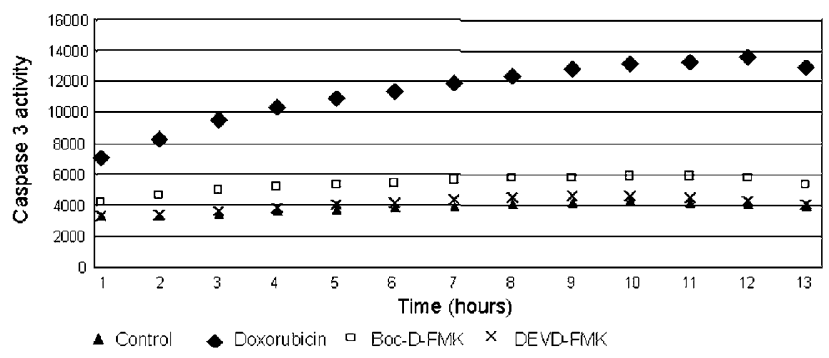

Figure 3 Caspase-3 activity as assessed in SU-DHL-1 cells treated with $5 \mu \mathrm{M}$ of doxorubicin for $6 \mathrm{~h}$. Treatment of SU-DHL-1 cells with doxorubicin resulted in significant increase of caspase-3 enzymatic activity, whereas the latter remained at the levels of control SU-DHL-1 cells after addition of either DEVD-FMK or Boc-D-FMK

compared with $1.2 \%$ in ALK-negative ALCL $(P=0.0003$ by Mann-Whitney test, Figure 5$)$.

BCL-2 was assessed in 49 ALCL $(20$ ALK +, 29 ALK-) tumors. BCL-2 was negative in all 20 ALKpositive tumors, but was positive in 21 of $29(72 \%)$ ALK-negative tumors $(P<0.0001$ by Fisher's exact test). The mean aC-3 level was $2.6 \%$ in BCL-2negative tumors compared with $1.1 \%$ in BCL-2positive tumors ( $P=0.01$ by Mann-Whitney test).

\section{Association of aC-3 Level with Apoptosis and Proliferation Index}

Tumors with high levels (higher than median) of aC-3 tended to have a higher apoptotic rate, but this association was not statistically significant $(P=0.3$ 

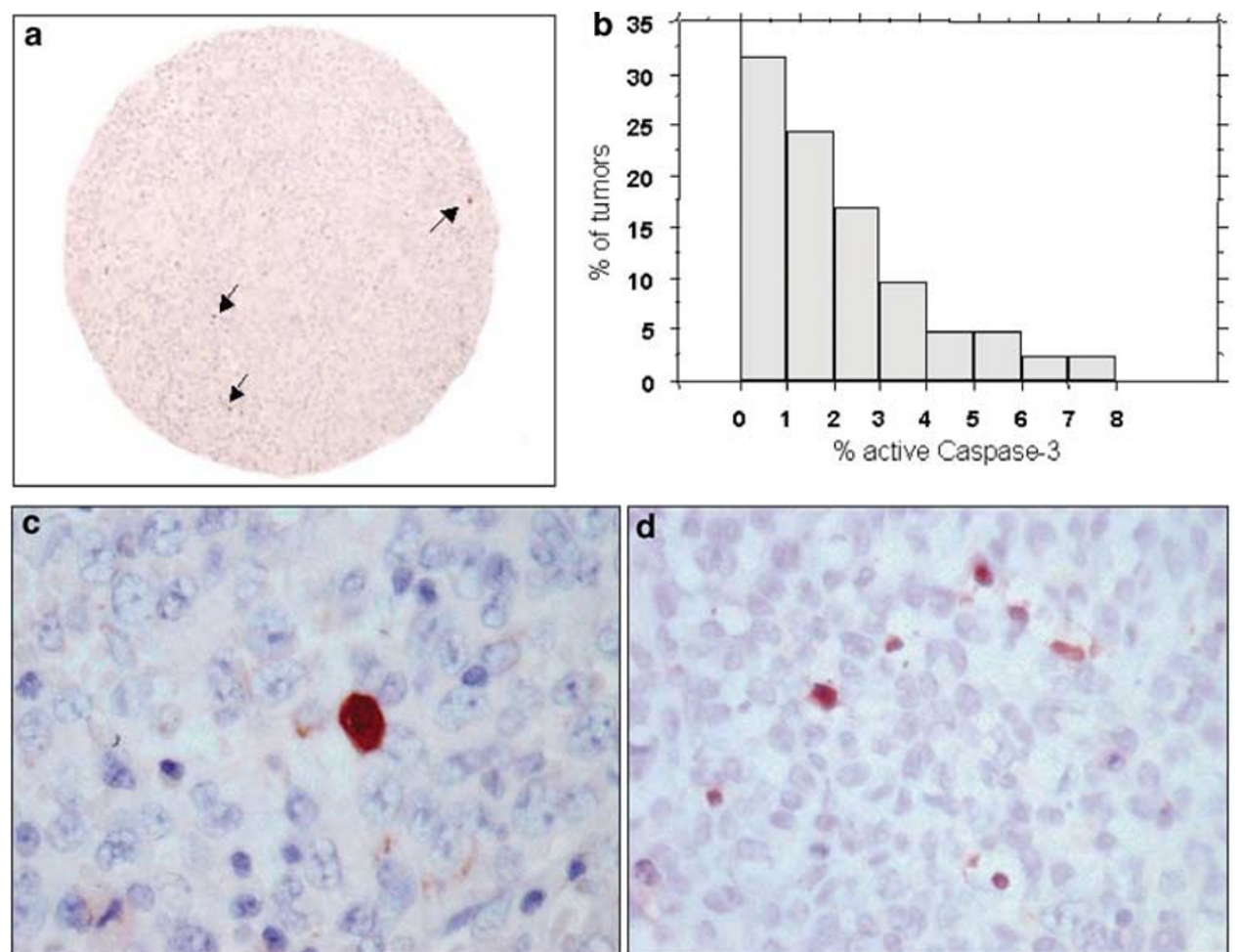

Figure 4 Immunohistochemical detection of activated caspase-3 (aC-3) in ALCL tumors: (a) representative tumor core from the ALCL tissue microarray. Arrows show aC-3 positive tumor nuclei. (b) distribution of ALCL tumors according to aC-3 levels; (c) an ALK-negative ALCL with low aC-3 expression levels; (d) an ALK-positive ALCL with high aC-3 expression levels (original magnification $\times 400$ for panel $\mathbf{c}$ and $\times 200$ for panel $\mathbf{d}$. DAB, hematoxylin counterstain, for panels $\mathbf{c}$ and $\mathbf{d}$ ).

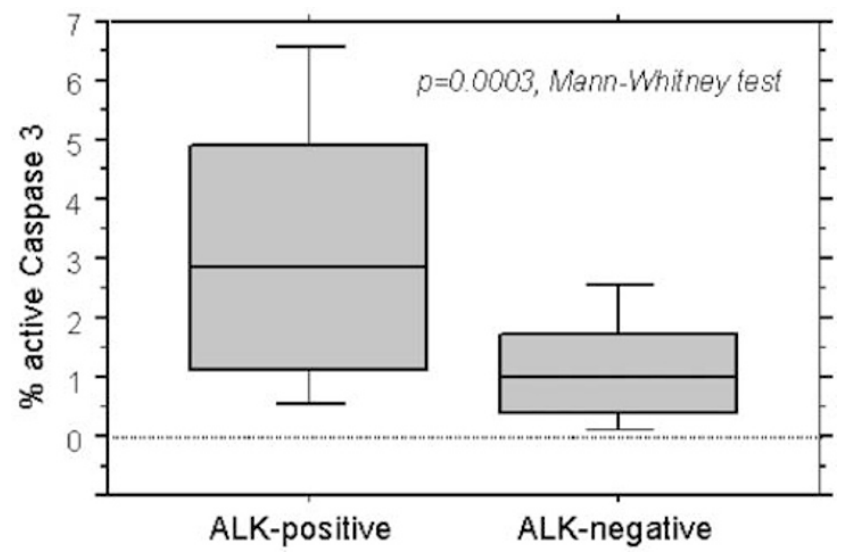

Figure 5 Box plot showing the significant difference in activated caspase-3 expression levels between ALK-positive and ALKnegative ALCL tumors.

by Mann-Whitney test). Proliferation index did not correlate with aC-3 level.

\section{Expression of proC-3 in ALCL Tumors}

The majority of germinal center cells in two reactive lymph nodes expressed proC-3 in a diffuse cytoplasmic pattern, as has been reported by others. ${ }^{25,26}$ proC-3, assessed in 36 ALCL (12 ALK+, 24 ALK-), was detected in all tumors. proC-3 expression was predominantly localized in the cytoplasm with less nuclear localization (Figure 6). Compared with reactive germinal centers, in ALCL proC-3 immunostaining was reduced in five $(14 \%$, two ALKpositive, three ALK-negative), preserved in 23 (64\%, five ALK-positive, 18 ALK-negative) and increased in eight $(22 \%$, five ALK-positive, three ALK-negative) tumors (Figure 6).

\section{Clinical Outcome}

Survival analysis included 51 ALCL cases with aC-3 results and follow-up. In 21 ALK-positive ALCL, we used the median of $2.9 \%$ aC-3 positive cells as a cutoff for high vs low aC-3 levels. The 5-year PFS was $81 \%$ for patients whose ALCL had high aC-3 levels vs $67 \%$ for patients whose ALCL had low aC-3 levels $(P=0.7$ by logrank). Similarly, OS did not correlate with aC-3 levels in the ALK-positive ALCL group.

In 30 ALK-negative ALCL, we used the median of $0.8 \%$ aC-3 positive cells as a cutoff for high vs low aC-3 levels. The 5-year PFS was $69 \%$ for patients with ALCL that had high aC-3 levels vs $43 \%$ for patients with ALCL that had low aC-3 levels $(P=0.3$ by logrank). Similarly, OS did not correlate significantly with aC-3 levels in the ALK-negative ALCL group. 

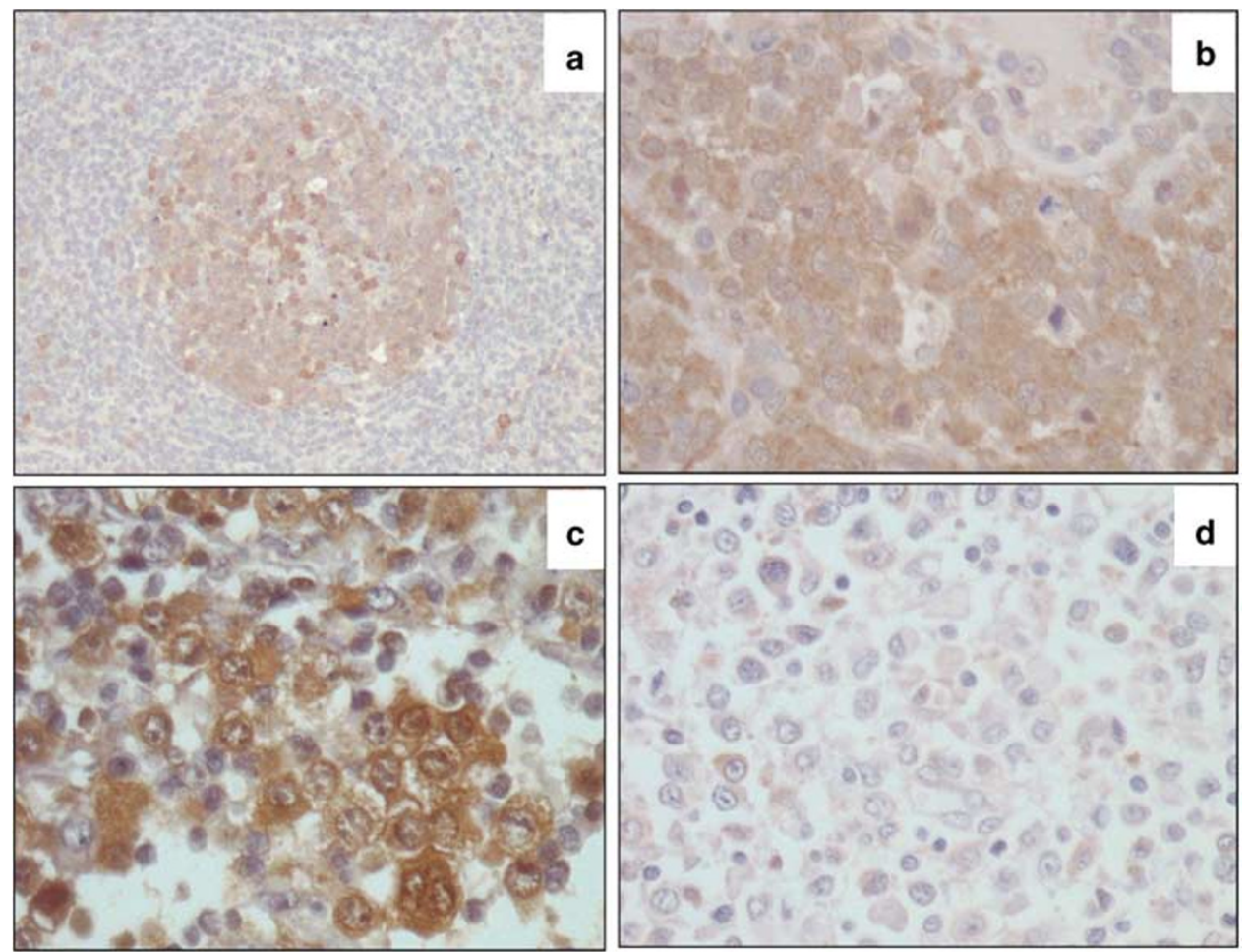

Figure 6 Immunohistochemical detection of procaspase-3 (proC-3) in reactive lymph node and ALCL tumors: (a) distribution of proC-3 expression in a reactive lymphoid follicle; (b) preserved proC-3 expression in ALCL; (c) increased proC-3 expression in ALCL; (d) reduced proC-3 expression in ALCL (DAB, hematoxylin counterstain, for all panels).

\section{Discussion}

A large body of evidence suggests that apoptotic cell death is ultimately mediated by caspase-3 in normal and tumor cells, but the exact mechanisms of caspase-3 activation and inhibition are currently under investigation. ${ }^{31}$ In this study, we report that doxorubicin-associated cell death involves caspase3 activation in two well-characterized ALCL cell lines, both of which carry the $t(2 ; 5)$ and overexpress ALK. We also demonstrate that caspase-3 activity was significantly elevated after treatment of cells with doxorubicin but remained at control levels when the caspase inhibitors were added. Proteolytic activation of caspase-3 may result from either extrinsic apoptotic signals, such as CD95L (FasL), after binding to specific receptors (CD95, Fas), or intrinsic apoptotic signals from mitochondria, such as expression of BCL-2 family members that can lead to activation of caspase-9 and release of cytochrome $c .^{22}$ Previous in vitro studies have shown that the CD95/CD95L pathway is active in ALCL cells. ${ }^{32,33}$ Furthermore, overexpression of BCL-2 family proteins $^{13,34}$ may also contribute to caspase-3-mediated cell death in ALK-positive ALCL.

We also report that expression levels of aC-3, an important executioner of apoptotic cell death, are significantly higher in ALK-positive than in ALK-negative ALCL tumors. This is in line with our previous studies that showed that apoptotic rate, as assessed by TUNEL, is significantly higher in ALK-positive tumors compared with ALKnegative ALCLs ${ }^{13}$ or other PTCLs. ${ }^{30}$ This difference may be due to differential expression of BCL-2 in ALK-positive ALCL and ALK-negative ALCL, as has been reported, ${ }^{13,35}$ or to other unknown factors. In a recent study, ten Berg et al ${ }^{36}$ also reported that high aC-3 levels correlate with ALK expression in systemic ALCL tumors. In that study, aC-3 was detected in a relatively larger percentage of ALCL tumor cells compared with the present report, but this difference may be due to the method of evaluation as they used an image analysis system. Although NPM-ALK is associated with antiapoptotic effects in vitro, ${ }^{15,16}$ it seems that ALKpositive ALCL is associated with a relatively increased apoptosis in vivo.

Levels of aC-3 in ALCL were associated with younger patient age in this study, most likely because higher aC-3 levels correlate with ALK expression, more frequent in younger patients with ALCL (see Materials and methods). There was a slight trend towards favorable PFS and OS for patients with ALCL tumors expressing high levels of aC-3 in this study group, but this association was not statistically significant. In contrast, ten Berg et $a l^{36}$ reported that expression of high levels of aC-3 significantly correlated with better OS in ALCL patients. However, the authors did not provide PFS data, which is not affected by variable postrelapse 
therapy. In addition, high aC-3 levels were not found to be an independent prognostic factor by multivariate analysis in that study. ${ }^{36}$

We also assessed proC-3 expression in a subset of 43 ALCL tumors and observed that the levels of the proenzyme are preserved or even increased in most ALCL tumors. Less than $15 \%$ of ALCLs showed reduced expression of proC-3, in agreement with previous reports. ${ }^{25,36}$ In a previous study, Donoghue et $a l^{26}$ correlated the pattern of proC-3 expression with prognosis in patients with diffuse large cell lymphoma, with the granular pattern correlating with better survival. ${ }^{26}$ We attempted this correlation but we found that distinguishing between expression patterns can be difficult using immunohistochemical methods and, not uncommonly, both patterns coexisted in an individual tumor.

In conclusion, we have shown that doxorubicininduced apoptotic cell death involves caspase-3 activation in ALK-positive ALCL cell lines. In addition, caspase- 3 activation positively correlates with ALK and inversely correlates with BCL-2 expression in ALCL tumors. Modulation of several factors involved in caspase-3 activation ${ }^{31}$ may provide novel targets for experimental therapy in ALCL patients.

\section{Acknowledgements}

Dr GZ Rassidakis is a recipient of an Alexander S Onassis Foundation scholarship.

\section{References}

1 Delsol G, Ralfkiaer E, Stein H, et al. Anaplastic large cell lymphoma. In: Jaffe ES, Harris NL, Stein H, Vardiman JW (eds). Pathology and Genetics of Tumors of Haematopoietic and Lymphoid Tissues. IARC Press: Lyon, France, 2001, pp 230-235.

2 Morris SW, Kirstein MN, Valentine MB, et al. Fusion of a kinase gene, ALK, to a nucleolar protein gene, NPM, in non-Hodgkin's lymphoma. Science 1994;263:12811284.

3 Lamant L, Dastugue N, Pulford K, et al. A new fusion gene, TPM3-ALK, in anaplastic large cell lymphoma created by a $(1 ; 2)(q 25 ; p 23)$ translocation. Blood 1999;93:3088-3095.

4 Hernandez L, Pinyol M, Hernandez S, et al. TRK-fused gene (TFG) is a new partner of ALK in anaplastic large cell lymphoma producing two structurally different TFG-ALK translocations. Blood 1999;94:3265-3268.

5 Ma Z, Cools J, Marynen P, et al. Inv(2)(p23q35) in anaplastic large-cell lymphoma induces constitutive anaplastic lymphoma kinase (ALK) tyrosine kinase activation by fusion to ATIC, an enzyme involved in purine nucleotide biosynthesis. Blood 2000;95:21442149.

6 Touriol C, Greenland C, Lamant L, et al. Further demonstration of the diversity of chromosomal changes involving 2p23 in ALK-positive lymphoma: 2 cases expressing ALK kinase fused to CLTCL (clathrin chain polypeptide-like). Blood 2000;95: 3204-3207.

7 Morris SW, Naeve C, Mathew P, et al. ALK, the chromosome 2 gene locus altered by the $t(2 ; 5)$ in non-Hodgkin's lymphoma, encodes a novel neural receptor tyrosine kinase that is highly related to leukocyte tyrosine kinase (LTK). Oncogene 1997;14: 2175-2188.

8 Fujimoto J, Shiota M, Iwahara T, et al. Characterization of the transforming activity of p80, a hyperphosphorylated protein in a Ki-1 lymphoma cell line with chromosomal translocation $\mathrm{t}(2 ; 5)$. Proc Natl Acad Sci USA 1996;93:4181-4186.

9 Bai RY, Dieter P, Peschel C, et al. Nucleophosminanaplastic lymphoma kinase of large-cell anaplastic lymphoma is a constitutively active tyrosine kinase that utilizes phospholipase C-gamma to mediate its mitogenicity. Mol Cell Biol 1998;18:6951-6961.

10 Falini B, Pileri S, Zinzani PL, et al. ALK+ lymphoma: Clinico-path findings and outcome. Blood 1999;93: 2697-2706.

11 Shiota $\mathrm{M}$, Nakamura $\mathrm{S}$, Ichinohasama $\mathrm{R}$, et al. Anaplastic large cell lymphomas expressing the novel chimeric protein p80NPM/ALK: a distinct clinicopathologic entity. Blood 1995;86:1954-1960.

12 Gascoyne RD, Aoun $\mathrm{P}$, Wu D, et al. Prognostic significance of anaplastic lymphoma kinase (ALK) protein expression in adults with anaplastic large cell lymphoma. Blood 1999;93:3913-3921.

13 Rassidakis GZ, Sarris AH, Herling M, et al. Differential expression of BCL-2 family proteins in ALK-positive and ALK-negative anaplastic large cell lymphoma of T/null-cell lineage. Am J Pathol 2001;159:527-535.

14 Duyster J, Bai RY, Morris SW. Translocations involving anaplastic lymphoma kinase (ALK). Oncogene 2001;20: 5623-5637.

15 Bai RY, Ouyang T, Miething C, et al. Nucleophosminanaplastic lymphoma kinase associated with anaplastic large-cell lymphoma activates the phosphatidylinositol 3-kinase/Akt antiapoptotic signaling pathway. Blood 2000;96:4319-4327.

16 Slupianek A, Nieborowska-Skorska M, Hoser G, et al. Role of phosphatidylinositol 3-kinase-Akt pathway in nucleophosmin/anaplastic lymphoma kinase-mediated lymphomagenesis. Cancer Res 2001;61:2194-2199.

17 Nieborowska-Skorska M, Slupianek A, Xue L, et al. Role of signal transducer and activator of transcription 5 in nucleophosmin/anaplastic lymphoma kinasemediated malignant transformation of lymphoid cells. Cancer Res 2001;61:6517-6523.

18 Zhang Q, Raghunath PN, Xue L, et al. Multilevel dysregulation of STAT3 activation in anaplastic lymphoma kinase-positive T/null-cell lymphoma. J Immunol 2002;168:466-474.

19 Zamo A, Chiarle R, Piva R, et al. Anaplastic lymphoma kinase (ALK) activates Stat3 and protects hematopoietic cells from cell death. Oncogene 2002;21:1038-1047.

20 Earnshaw WC, Martins LM, Kaufmann SH. Mammalian caspases: structure, activation, substrates, and functions during apoptosis. Annu Rev Biochem 1999;68:383-424.

21 Schlegel J, Peters I, Orrenius S, et al. CPP32/apopain is a key interleukin 1 beta converting enzyme-like protease involved in Fas-mediated apoptosis. J Biol Chem 1996;271:1841-1844. 
22 Hengartner MO. The biochemistry of apoptosis. Nature 2000;407:770-776.

23 Woo M, Hakem R, Soengas MS, et al. Essential contribution of caspase 3/CPP32 to apoptosis and its associated nuclear changes. Genes Dev 1998;12: 806-819.

24 Chhanabhai M, Krajewski S, Krajewska M, et al. Immunohistochemical analysis of interleukin-1betaconverting enzyme/Ced- 3 family protease, CPP32/ Yama/Caspase-3, in Hodgkin's disease. Blood 1997;90:2451-2455.

25 Krajewski S, Gascoyne RD, Zapata JM, et al. Immunolocalization of the ICE/Ced-3-family protease, CPP32 (Caspase-3), in non-Hodgkin's lymphomas, chronic lymphocytic leukemias, and reactive lymph nodes. Blood 1997;89:3817-3825.

26 Donoghue S, Baden HS, Lauder I, et al. Immunohistochemical localization of caspase-3 correlates with clinical outcome in B-cell diffuse large-cell lymphoma. Cancer Res 1999;59:5386-5391.

27 Dukers DF, Oudejans JJ, Vos W, et al. Apoptosis in Bcell lymphomas and reactive lymphoid tissues always involves activation of caspase 3 as determined by a new in situ detection method. J Pathol 2002;196: 307-315.

28 Mancini M, Nicholson DW, Roy S, et al. The caspase-3 precursor has a cytosolic and mitochondrial distribution: implications for apoptotic signaling. J Cell Biol 1998;140:1485-1495.
29 Bradford MM. A rapid and sensitive method for the quantitation of microgram quantities of protein utilizing the principle of protein-dye binding. Anal Biochem 1976;72:248-254.

30 Rassidakis GZ, Jones D, Thomaides A, et al. Apoptotic rate in peripheral T-cell lymphomas. A study using a tissue microarray with validation on full tissue sections. Am J Clin Pathol 2002;118:328-334.

31 Shi Y. Mechanisms of caspase activation and inhibition during apoptosis. Mol Cell 2002;9:459-470.

32 Dirks W, Schone S, Uphoff C, et al. Expression and function of CD95 (FAS/APO-1) in leukaemia-lymphoma tumour lines. Br J Haematol 1997;96:584-593.

33 Greenland C, Touriol C, Chevillard G, et al. Expression of the oncogenic NPM-ALK chimeric protein in human lymphoid T-cells inhibits drug-induced, but not Fasinduced apoptosis. Oncogene 2001;20:7386-7397.

34 Rassidakis GZ, Lai R, McDonnell TJ, et al. Overexpression of mcl-1 in anaplastic large cell lymphoma cell lines and tumors. Am J Pathol 2002;160:23092310.

35 Rassidakis GZ, Jones D, Lai R, et al. BCL-2 family proteins in peripheral T-cell lymphomas: correlation with tumour apoptosis and proliferation. J Pathol 2003;200:240-248.

36 ten Berge RL, Meijer CJ, Dukers DF, et al. Expression levels of apoptosis-related proteins predict clinical outcome in anaplastic large cell lymphoma. Blood 2002;99:4540-4546. 